\title{
Medical Image Segmentation by Fish Schooling Algorithm and Neural Network
}

\author{
K V Sandeep ${ }^{1}$, Manoj Dandamudi ${ }^{1}$ and P Dhanusha ${ }^{1}$ \\ ${ }^{1}$ Ramaiah Institute of Technology, CSE Department, Bangalore, India
}

To Cite this Article

K V Sandeep, Manoj Dandamudi and P Dhanusha. Medical Image Segmentation by Fish Schooling Algorithm and Neural Network. International Journal for Modern Trends in Science and Technology 2021, 7, pp. 55-60. https://doi.org/10.46501/IJMTST0710009

\section{Article Info}

Received: 06 September 2021; Accepted: 30 September 2021; Published: 03 October 2021

\section{ABSTRACT}

Medical image diagnosis by machine decrease the doctor load and increases the efficiency of treatment as well. Many of diagnosis process depends on chemical data and some are depend on digital images. This work focus on brain tumor medical image diagnosis by segmenting the tumor region in the image. For tumor detection neural network was trained by the model. Selected features extract from the image by fish schooling genetic algorithm for training of neural network It was obtained that fish schooling based genetic feature selection has increases the detection accuracy of trained model. Experiment was done on real dataset and results compared with existing techniques of tumor detection from MRI images.

KEYWORDS: Image Segmentation, Machine Learning, Soft Computing, Genetic Algorithm.

\section{I.INTRODUCTION}

Biomedical image analysis helped doctors a lot in achieving perfect diagnosis [1] and it also helps several biologists in their discoveries $[2,3]$. Computer-aided biological discoveries help in achieving high-quality information such as information regarding tumors, the morphology of cells, quantity, glands, and density. It also offers a wide selection of images such as CT images, whole side tissue images, MR images, and many more.

A brain tumor is a condition when there is the formation of unusual cells in our brain. Such unusual cell affects the functioning of the brain and creates problems in the overall health of a person [4]. The main focus of several researchers and experts these days is to use the technique of medical imaging to study the brain of the patient so that it can be cured at an early stage as late detection of such cells has killed many patients in the entire world [5]. Till now detection of tumor cells in the brain of the patients is done manually by experts who take a lot amount of time. One more problem with this is the differences in the MR image obtained by different experts. Eve the same physician was found to have different sets of MR images which created confusion. Because of all the above reasons, it was important to develop an automatic detection process to detect the brain tumor to save time and to achieve 100\% accuracy.

The main problem in biomedical image analysis is the segmentation of the image. The process is to obtain the information from the input images and form a meaningful segment that can be recognized as a tumor. As soon as such results are obtained one can easily work on these affected cells and extract them from the brain. The tumor's size is calculated by the counting of pixels inside the affected area. Many approaches were 
proposed to achieve success and accuracy in this image segmentation process [9]. The study of image segmentation has been going through decades and is still in the process many of the experts have done deep learning methods and have developed powerful tools to achieve a higher level of accuracy.

\section{LITERATURE SURVEY}

Nilesh Bhaskarrao Bahadure et. al. [5] HE also made an attempt to solve the complexities-related problem that comes during the image segmentation process. He invented (Berkely Wavelet transformation) that helps to solve problems during the image segmentation process. He also proposed SVM(support vector machine) to improve the accuracy further to obtain better features from the segmented tissue.

Mohammed Sahib Mahdi Altaei et. al. [6] A SIFT descriptor was introduced by him to obtain MRI features or images of the brain to identify the tumor. An excellent brightened image was obtained by using this technique when the MRI image was rotated. A huge number of other features that were helpful for MRI classification were also obtained by this process. HE adopted 2two models to classify levels.

Ashish et. al. [7] Used ISBGO(Bio-geography Optimization) to obtain image segmentation and in this algorithm, every time accuracy of the random segment was reduced as compared with the same image set.

T. Chithambaram et. al. [8] He proposed ANN(Artificial Neural Network) which works in vector quantization manipulation process to conduct the automated MRI-scans in finding the tumors. Computational time, training performance, and classification accuracies were some of the parameters that were studied in this modified ANN. As there are many complexities that are associated with tumors is a tedious task to obtain the exact MRI images of the brain. Two techniques were introduced by him for such tumor detection-Histogram Thresholding, Artificial Neural Network technique. One important factor during the MRI process is to detect the edge segmentation of the tissue during brain scan and so an algorithm was proposed by him to solve the problem with segmentation.

Chenyu, et al. [9] discussed various techniques that were proposed earlier related to the reduction of noise during ionizing radiation. And then he proposed his own algorithm which can give high-quality images along with low dose and with improved noise reduction. He provided six different methods related to CT(LDCT). SMGAN-3d was also proposed by him which gives high-quality images than the present process.

Taghizadeh et. al. [10] Proposed that the direct use of stereo radio surgery ((SRS) on the patient causes a harmful and noisy impact on the body in form of ionizing radiation. His work revolves around within comparison of MRI sequence with SRS and CT technique.

\section{PROPOSED MODEL}

\section{Input Image De-Noising}

Input Image may have some noise in when transfer form analog to digital. So proposed model has utilized weiner filter for the de-noising of image. Image is blocked into sub blocks of bxb dimension and each block was processed to remove noise from image.

\section{Skull Removal}

In order to identify brain region of input image some more pre-processing steps need to be apply after de-noising. Skull region of image should be identify for this and remove. De-noised image is convert into binary image. For image conversion threshold method was used in the work as shown in eq. 1.

$$
\mathrm{E}=1 \text { if } \mathrm{F}>\mathrm{T} \text { or } \mathrm{E}=0 \text { if } \mathrm{F}<=\mathrm{T}-\mathrm{Eq} .1
$$

Inner white color pixel value was consider as brain region of image. Outer white pixel cell values were consider as skull part of image. Once marking of pixels having brain region was identified then image gray pixel values were reconsidered.

\section{Genetic Algorithm Fish Schooling}

Fish Population: GAFS have set of chromosomes that is set of pixel value consider as cluster center. Each element in pixel set is consider as cluster center representative in the work. A image was divide into tumor region and non tumor region in image. Each chromosome is acting as fish in the algorithm. Fish population is a matrix where rows represent chromosome and column represent cluster center. 


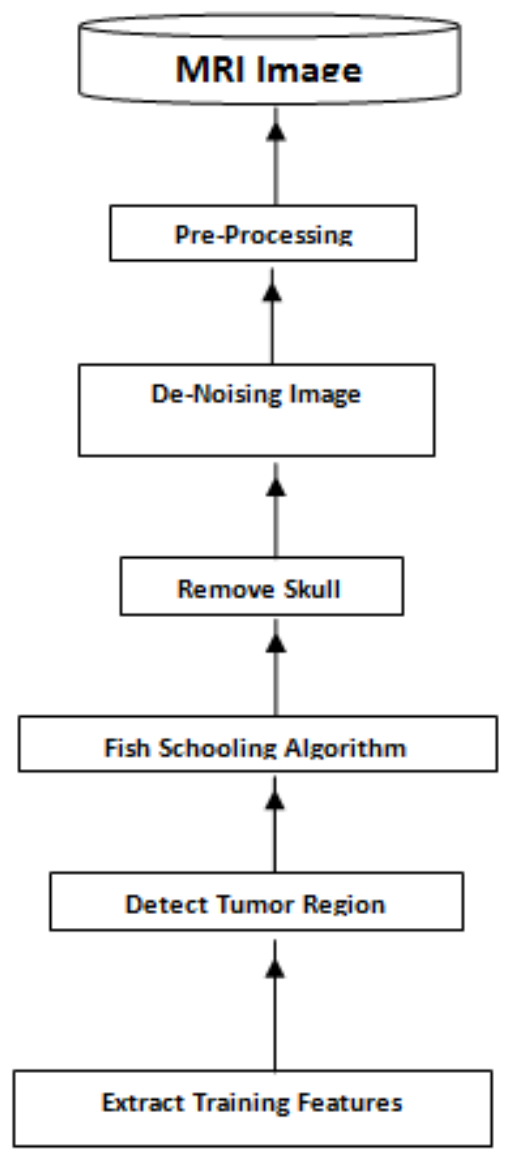

Fig. 1 Block diagram of work.

FP®GenerateFish(BD, n, m)-Eq. 2

FP matrix have $m$ number of rows and $n$ number of columns. BD is brain region gray image. Cluster centers were select randomly in the chromosomes.

\section{Fitness Function}

Fitness value of fish need to be calculate as per eq. 3 . Summation of distance between the cluster center and image gray pixel value of brain region is consider as fitness value of a chromosome. Difference was evaluate by Euclidian function

$$
F P_{s}-I(x, y) \vee
$$

$$
\begin{aligned}
& \min _{\sum_{1}^{y}} \\
& F_{m}=\sum_{1}^{x}
\end{aligned}
$$

In above equation $\mathrm{F}_{\mathrm{m}}$ is fitness value of $\mathrm{m}^{\text {th }}$ solution in the population. Input brain image after skull removal $I_{x y}$ have dimension $\mathrm{x}$ and $\mathrm{y}$. In this work each pixel value of image was subtract from the cluster center pixel values and minimum distance value was summed. Final summation of all pixel value was term as fitness value of $\mathrm{mth}$ chromosome in the population.

\section{Parameters}

$S_{\mathrm{vol}}$ : Maximum displacement perform in a operator.

$\mathbf{X}(\mathbf{t})$ : Random position of a document in the clusters for $\mathrm{t}^{\text {th }}$ iteration.

$\mathbf{W}(t)$ : Summation of fitness value.

1)

\section{2) 4.4.3 Collective volitive Movement}

All fishes either reduce there distance if barycenter $\mathrm{B}(\mathrm{t})$ is found otherwise dispersion movement is applied, so if sum of fitness weight SW value is higher than previous iteration than movement operation done by case1 otherwise case2 apply.

$$
\begin{aligned}
& S W=\sum W(t)+\Delta F \\
& \Delta F=W(t)-W(t-1)
\end{aligned}
$$

\section{Case1}

$$
x(t+1)=x(t)-S_{v o l} \times R \times\left(\frac{x(t)-B(t)}{\text { Euclidean }(x(t), B(t))}\right)
$$

\section{Case2}

$$
x(t+1)=x(t)+S_{\text {vol }} \times R \times\left(\frac{x(t)-B(t)}{\text { Euclidean }(x(t), B(t))}\right)
$$

In above eq. $\mathrm{R}$ is random number range between 0 to 1 . Initial value of Svol is cluster size or DS/number ofcluster.

$$
B(t)=\frac{\sum_{i=1}^{N} x(t) W(t)}{}
$$

\section{Feeding Operator}

$$
\begin{gathered}
\Delta f \vee \\
\max (\quad \mid) \\
\frac{\Delta f}{-} \\
W(t+1)=W(t)+ \\
S_{\text {vol }}=S_{\text {vol }}-\frac{S_{\text {vol }}}{\text { Iteration }_{\text {max }}}
\end{gathered}
$$

\section{Crossover}

In this work FP fish population were altered according to $x(t)$ values which range in 0 to greatest number of cluster center. Hybrid activity produce new cluster set chromosome values of pixel, while new fish is possibly 
acknowledge whether wellness esteem is acceptable than past.

\section{Population Update}

Crossover operation increase the number of population in the algorithm. This increase in number need to be adjust so this step of population updation. In this step new chromosome fitness value were calculate. If new chromosome fitness value is higher than than parent chromosome then parent chromosome was removed from the population otherwise childe or new chromosome removed. This selection or rejection of chromosome was population updation.

\section{Cluster Center}

So when above fitness value assessment crossover operation and population update done for $t$ number of cycles then final population best chromosome fist act as cluster center of image that cluster image. Based on the cluster center values image was cluster into tumor region that is infected portion of image and non tumor region that is brain healthy portion.

\section{Segmented Image}

Fish schooling genetic algorithm provide the cluster in the image as image was broadly segment into two region first is tumor part and other is non-tumor part. So two cluster center representing pixels were obtained by the proposed model FSGA. As per the Euclidian distance pixels are segment and give single color value for the group.

\section{Extract Training Feature}

Input cluster image is blocked in $3 \times 3$ matrix and each block is transform into vector of 9 values. As per block maximum pixel class count desired output value 1 set (tumor region) and 0 for (Non-tumor region). Once training vector ready with input feature set and desired output set, feed forward neural network is used for learning of classified data. Output of this step is trained neural network that takes $3 \times 3 \times$ block of image and gives class of tumor or non tumor.

\section{EXPERIMENT SETUP AND RESULTS}

Experiment work was done on machine having hardware configuration of $i 3$ processor $6^{\text {th }}$ generation and 4 GB RAM. MATLAB software was used for the work and implementation was done on MATLAB editor of 2016 version. Proposed work was compared with previous work done in [13].

\section{Dataset}

In order to conduct the experiment an real dataset which is a collection of images from different category are utilize.
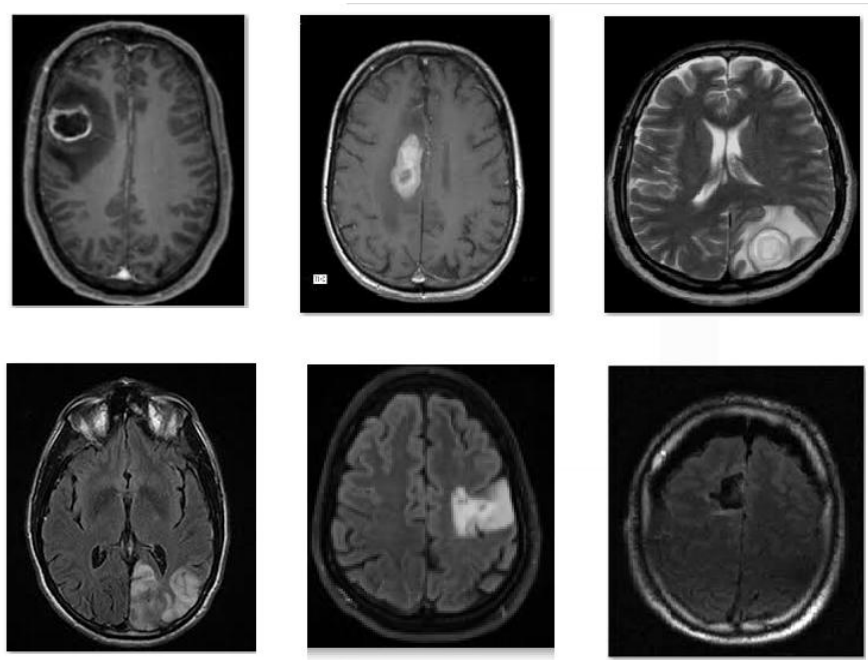

Table 1 Dataset of Different category.

As images are of different format so first it is necessary to make it in readable format for experiment tool MATLAB. Now this collection of images of different category is shown in table 1.

\section{RESULT}

Table 2 Precision value based comparison of proposed model.

\begin{tabular}{|l|l|l|}
\hline Image & Proposed Model & Previous Model \\
\hline Set 1 & 0.916 & 0.9069 \\
\hline Set 2 & 0.9829 & 0.9823 \\
\hline Set 3 & 0.9725 & 0.9725 \\
\hline Set4 & 0.9944 & 0.9927 \\
\hline
\end{tabular}

Table 2 shows that proposed model has increase the precision value of tumor detection as compared to previous model proposed in [13]. It was also shown that proposed model filtration method has increase the segmentation accuracy. Use of fish schooling genetic algorithm for tumor cluster detection has increase the precision value as well. 
Table 3 Recall value based comparison of proposed model.

\begin{tabular}{|l|l|l|}
\hline Image & Proposed Model & Previous Model \\
\hline Set 1 & 0.9881 & 0.8903 \\
\hline Set 2 & 0.9971 & 0.992629 \\
\hline Set 3 & 0.9662 & 0.9168 \\
\hline Set 4 & 0.9925 & 0.9863 \\
\hline
\end{tabular}

Table 3 shows that finding of tumor pixel in input image was more effectively identify by the proposed genetic algorithm. Use of trained navies bayes classifier was static for all set of testing image. Proposed fish schooling genetic algorithm has find tumor representative cluster center pixel dynamically for each input image. Hence proposed model recall values are increased by dynamic cluster center approach.

\section{Table 4 F-measure value based comparison of} proposed model.

\begin{tabular}{|l|l|l|}
\hline Image & Proposed Model & Previous Model \\
\hline Set 1 & 0.9507 & 0.8985 \\
\hline Set 2 & 0.9899 & 0.987449 \\
\hline Set 3 & 0.9694 & 0.9438 \\
\hline Set4 & 0.9932 & 0.9895 \\
\hline
\end{tabular}

Table 4 shows that proposed model has increase the precision value of tumor detection as compared to previous model proposed in [13]. It was also shown that proposed model filtration method has increase the segmentation accuracy. Use of fish schooling genetic algorithm for tumor cluster detection has increase the precision value as well.

Table 5 Accuracy value based comparison of proposed model.

\begin{tabular}{|l|l|l|}
\hline Image & Proposed Model & Previous Model \\
\hline Set 1 & 90.6546 & 81.669 \\
\hline Set 2 & 98.0116 & 97.5256 \\
\hline Set 3 & 94.0917 & 89.4458 \\
\hline Set4 & 98.6649 & 97.9296 \\
\hline
\end{tabular}

Table 5 shows that finding of tumor pixel in input image was more effectively identify by the proposed genetic algorithm. Use of trained navies bayes classifier was static for all set of testing image. Proposed fish schooling genetic algorithm has find tumor representative cluster center pixel dynamically for each input image. Hence proposed model recall values are increased by dynamic cluster center approach.

\section{CONCLUSIONS}

As the tumor segmentation assumes critical part in brain tumor treatment. So proposed strategy use the best possible filtration way to deal with section the MRI picture of brain tumor into tumor and non tumor area. Medical diagnosis depend on testing equipment, pathology tests, and doctor experience. So this work has proposed FSGA a model for brain tumor detection in MRI images. Input brain MRI image skull pixels were also identified and removed in the work to reduce the confusion in image segmentation. It is obtained that proposed fish schooling genetic algorithm and neural network ssegments the image with high accuracy. This work has increased the accuracy of the segmentation so the medical diagnosis gets easy and fast. Here overall precision and recall values are also good from segmentation view.

\section{REFERENCES}

[1] Astina Minz, Prof. Chanddrakant Mahobiya "MR Image classification using Adaboost for brain tumor type" in IEEE 7th International Advance Computing Conference (IACC) 2017.

[2] Garima Singh, Dr. M. A. Ansari “Efficient Detection of Brain Tumor from MRIs Using K-Means Segmentation and Normalized Histogram" in IEEE Issue 2016.

[3] Zehra Karhan, Burhan Ergen.(2015). Content Based Medical Image Classification Using Discrete Wavelet and Cosine Transforms. IEEE.

[4] Parveen, Amritpal Singh.(2015). Detection of Brain Tumor in MRI Images, using Combination of Fuzzy C-Means and SVM. 2nd International Conference on Signal Processing and Integrated Networks (SPIN).

[5] Nilesh Bhaskarrao Bahadure, Arun Kumar Ray, and Har Pal Thethi. "Image Analysis for MRI Based Brain Tumor Detection and Feature Extraction Using Biologically Inspired BWT and SVM" international journal of biomedical imaging hindwai, 2017.

[6] Mohammed Sahib Mahdi Altaei and Sura Yarub Kamil. "Brain tumor detection and classification using SIFT in MRI images" AIP Conference Proceedings 2292, 2020.

[7] Ashish Dehariya and Dr. Pragya Shukla, " Brain Cancer Prediction Through Segmentation Of Images Using Bio-Geography Based Optimization". International Journal of Advanced Research in Engineering and Technology (IJARET), Volume 11, Issue 11, (November 2020)

[8] T. Chithambaram, K. Perumal. "Edge Detection Algorithms Using Brain Tumor Detection and Segmentation Using Artificial Neural Network Techniques". International Research Journal of 
Advanced Engineering and Science, Volume 1, Issue 3, pp. 135-140, 2016.

[9] Yu Chenyu "Structurally-sensitive multi-scale deep neural network for low-dose CT denoising." IEEE Access 6 (2018): 41839- 41855.

[10] Taghizadeh, Somayeh, et al. "Optimizing MRI sequences and images for MRI-based stereotactic radiosurgery treatment planning." Reports of Practical Oncology \& Radiotherapy 24.1 (2019): 12-19.

[11] Dr.Samir Kumar Bandhyopadhyay, Tuhin Utsab Paul," Segmentation of Brain MRI Image - A Review", International Journal of Advanced Research in Computer Science and Software Engineering ISSN: 2277 128X, Volume 2, Issue 3, March 2012.

[12] V. Anitha, S. Murugavalli. "Brain tumour classification using two-tier classifier with adaptive segmentation technique". ISSN 1751-9632, IET on 22nd June 2015.

[13] Hein Tun Zaw, Noppadol Maneerat, Khin Yadanar Win. "Brain tumor detection based on Naïve Bayes Classification". 2019 IEEE

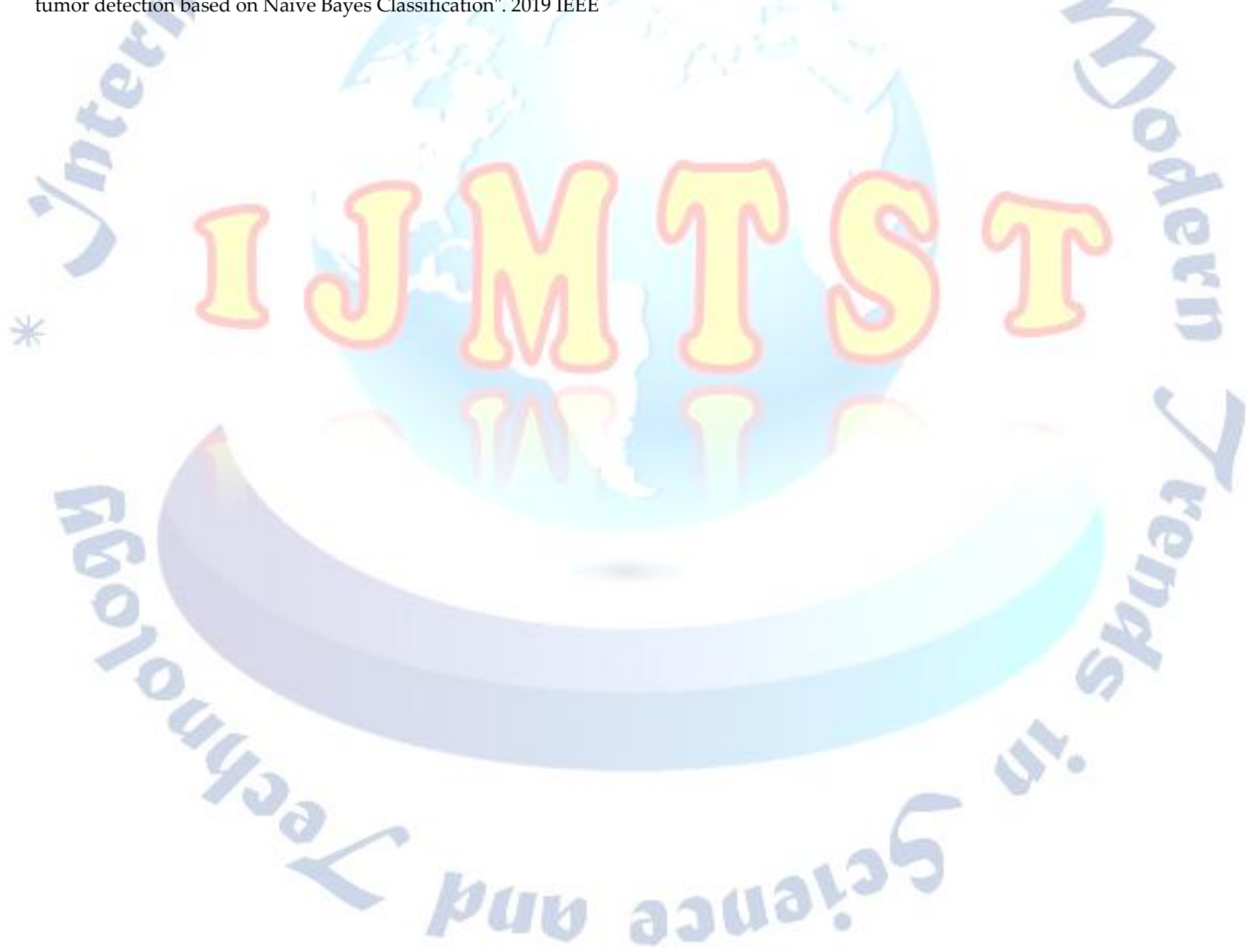

